\title{
PREFACE \\ A SPECIAL SELECTION ON MECHANICAL ENGINEERING APPLIED TO BIOMEDICINE — PART II
}

\author{
Published 16 December 2020
}

Part II of this special issue collects the contributions concentrating on Mechanical Engineering Applied to Biomedicine. It aims to accounts for outstanding and novel research in mechanics used for biomedicine and relevant fields. The details of the accepted 23 papers are given as follows:

Yeon Wook Kim et al. propose a new auto-scoring algorithm that automatically evaluates the Berg balance scale (BBS) tasks. The BBS can be used as an indicator for patients to analyze their rehabilitation status by themselves. They, after applying the BBS auto-scoring algorithm for wearable IMU sensors, conclude that patients can always evaluate their movements. Through this process, the patient will be able to practice rehabilitation steadily in daily life and the effectiveness of rehabilitation will be maximized.

Xinhua $\mathrm{Hu}$ et al. discuss the difficulties and challenges of the application of the functional near-infrared spectroscopy (fINRS) currently experienced in various neurological diseases, such as Parkinson's disease, epilepsy and stroke. In this emerging field, many of its complementary technologies such as EEG and BCI are also promising. They conclude that fNIRS can be developed into a low-cost option that would widely permit us to diagnose brain disorders using a more objective method based on brain activity and also to monitor the efficacy of therapeutic approaches during the course of treatment.

Lung-Fa Pan et al. analyze that the Taguchi dynamic analysis and the orthogonal array re-organizing the essential factors are crucial for the optimization of the TLD readout system given strict criteria of multiple irradiated environments and long-term exposure for calibrated thermoluminescent dosimeters (TLDs). They conclude that the TLD fading as one more factor influencing the TLD response curve will be added in the multifactor analysis covering five factors and Taguchi analysis of four factors, including the TLD fading and excluding one minor factor (preheat temperature of maximal temperature).

Xiaozhao Chen et al. put forward quantitative assessments for the firmness of pedicle screws taking bone mass as the basis. Quantitative assessments of the 
firmness of screw trajectories are made by computing the total mineral content of the bone supporting screws. They conclude that the method proposed not only guides doctors to choose a firmer screw channel but also help them predict the postoperative firmness and prevent issues such as falling off of screws due to the low value of a patient's bone mass.

Do Yeon Kim et al. propose a method that the displacement amplification ratio is derived using the formula of a bridge-type structure, and the displacement and resonance frequency are derived by finite element analysis (FEA) using different variables. They propose a piezoelectric actuator with a bridge structure inserted into the ear canal. The piezoelectric actuator is fabricated on the basis of FEA simulation results and verified through an artificial mastoid for stimulation in the ear canal. They conclude that the most efficient thickness and material are selected, and it is confirmed that the efficiency increased through a comparison experiment with an existing piezoelectric body using LDV.

Yushu Liu et al. work on understanding the relationship between the changes in the axonal transport of the optic nerve and lamina cribrosa (LC) deformation, and they answer if this will be helpful to estimate the degree of axonal transport block by measuring the LC deformation in vivo. The results show axonal transport changes of ON increased with elevation of the high intraocular pressure (IOPs). They conclude in the present experiment and mechanical analysis of the finite element model both serve as a reasonable explanation of the hypothesis of pressure-dependent optic nerve $(\mathrm{ON})$ damage in glaucoma.

Yong-Un Jo et al. aim to robustly recognize hand gestures in real-time using convolutional recurrent neural network (CRNN) with pre-processing and overlapping windows. They conclude that according to the results of these experiments, it is possible to classify normally only when EMG data over a certain period $(250 \mathrm{~ms})$ is received in all tests. It is shown that learning the data of all subjects had lower test performance than learning individual data separately. This is a problem that can be caused by various factors and is expected to occur due to the large individual differences in the EMG pattern.

Jin Woong Yi et al. confirm the effective insertion angle $\left(0^{\circ}, 30^{\circ}, 50^{\circ}\right.$, and $\left.90^{\circ}\right)$ of the fibular allografts (FA). They apply axial and shear loads to finite element models used in their study. They evaluate the construct stiffness, stress distribution on the plate and fibula, and fracture micromotion. The result shows that vertically inserted FA contributes minimally than other insertion angle for preventing secondary complications such as varus collapse of the humeral head. Thus, horizontal insertion of the FA would be more effective than the vertical insertion method during the actual surgery.

Ji-Ming Ying et al. use a portable biofeedback-based gait training device to advocate able-bodied subjects to adopt different walking strategies in order to alter the plantar pressure distribution during walking. This could potentially be useful to guide diabetic patients to perform gait retraining for pressure reduction purposes. 
They conclude that the relationship between joint coordination and pressure redistribution pattern could potentially be useful to guide diabetic patients to perform gait retraining for pressure reduction purposes.

Bum-Suk Lee et al. work on tests and validate a usability scale for power wheelchairs. As a result, they conclude that the usability scale for power wheelchairs may be very useful to evaluate the overall usability of power wheelchair including its safety, operability, efficiency, and satisfaction. The developed usability scale (USWP) may help wheelchair makers become aware of the problems of present power wheelchairs and the users' needs, allowing them to make better ones. It is expected that the quality of domestic power wheelchair products can be improved through the developed usability scale.

Won-Seuk Choi et al. aim to develop the adjustable curb and stairs as wheelchair training structures and verify their usefulness. They conclude that training using this type of wheelchair will help wheelchair users improve the confidence and skills of using a wheelchair which are essential for their safety and independent mobility. The results of this study may be useful in developing and evaluating various assistive devices and products for the disabled as well as wheelchair training structures.

Byung-Woo Ko et al. study the changes in gait symmetry with trunk displacement during phase-shifted auditory-paced treadmill walking for effective training with auditory cueing provided in conventional gait training. The results indicate that the phase-shifted auditory cue significantly changes gait symmetry and trunk displacement. In the future, these clinical results will be progressively evaluated with stroke survivors using a similar concept. This approach could be applied in the development of an auditory cueing system that can provide real-time feedback. If a patient uses a phase-shifted auditory cueing system in daily life, the patient could undergo training for symmetrical gait with suitable trunk displacement.

Kai-Yu Hsiao et al. investigate the overall survival prediction for non-small cell lung carcinoma (NSCLC) patients of clinical IIIA-N2 stage undergone various treatments through a refined Taylor series expansion algorithm. The proposed prediction algorithm is based on the well-known hit and target model adopted for analyzing cell death from the microscopic viewpoint. In the proposed algorithm, the basic degradation of a patient's health is represented via a specific function comprising a single exponential term $\exp (-\alpha \mathrm{t})$. The refined algorithm successfully predicts NSCLC IIIA-N2 patients' overall survival rate. An index of life gain with specific treatment is defined according to the Taguchi optimization analysis.

Wan-Ho Jang et al. compare an off-loading cushion, designed for individuals with spinal cord injury, with an air cushion to analyze the effect of pressure on skin injury and user satisfaction. The off-loading cushion can reduce the incidence of pressure ulcers by minimizing the pressure of the ischial tuberosity and coccyx. In the 3D modeling, the product is designed so that the ischial tuberosity and coccyx have 
minimal contact with the cushion's surface area. This study suggests that offloading cushions reduce the occurrence of pressure injury compared to air cushions.

SeonHong Hwang et al. investigate the quantitative scaling properties of the center of pressure (COP) as well as the spatial-temporal properties of the COP to elucidate the postural control behavior of healthy elderly (HE) adults and adults with Parkinson's disease (PD) during quiet standing. They conclude the results of this study indicate that it may be possible to improve COP control through specific functional training on visual and proprioceptive components of balance rehabilitation for people with PD.

Yan-Lin Wang et al. use the bionic muscle cable to replace the ordinary cable in the cable-driven parallel robots (CDPR), which can solve the pseudo-drag phenomenon of the cables in the CDPR and improve the safety performance of the rehabilitation robot. The influence of the parameters of the elastic elements in the bionic muscle cables on the mechanical characteristics of the bionic muscle cabledriven lower limb rehabilitation robot (BMCDLR) system is analyzed by using simulation experiments. The research results can provide a reference basis for research on the safety evaluation and control methods of the BMCDLR system.

Jing Li et al. report the design, fabrication and calibration results of a miniature cross-shaped three-axial piezoresistive force sensor, which can simultaneously detect three force components in orthogonal directions. MEMS technology is used to fabricate the sensor structure and deposit a phosphosilicate layer on the silicon wafer to form piezoresistive resistors. Using the finite element simulation, the developed sensor performance characteristics. Further optimization of the sensor design for sensing six degrees of freedom movement is envisaged with its sensitivity enhancement by the silicon substrate reduction.

Sang-Hong Lee et al. propose a new instance selection method that combines the neural network with weighted fuzzy memberships (NEWFM) and Takagi-Sugeno (T-S) fuzzy model is proposed to improve the classification performance of healthy people and Parkinson's disease (PD) patients. The study shows that NEWFM with instance selection performed better than NEWFM without instance selection. In addition, when a Mcnemar's test is performed to determine the difference in performance before and after instance selection, the probability of significance is determined to be 0.031 .

Yong Cheol Kim et al. design and validate a new bilateral instrumented wheel system to measure triaxial handrim forces and torques simultaneously during wheelchair propulsion. The designed and implemented system measures the force applied to the handrims on both sides of a manual wheelchair using 6-axis force/ torque sensors. They conduct static and dynamic tests in which the developed bilateral IWS is used to measure the forces applied to the handrim and evaluate the measurement accuracy. In future work, they will conduct clinical research using the developed IWS, and combine the IWS with a motion analysis system to perform further research on manual wheelchairs. 
Kyong Kim et al. present a study that intends to develop a system for managing the health of the spinal joints of elderly people who mainly live on Korean floor heating. They develop a posture balance device which detects the center of pressure (COP) of the spine and the direction of the upper body movement, and a spine health management software featuring a game based on the collected data. The posture balance device is small in volume, and excellent in utilization, and it is expected to help patients with spinal diseases perform corrective exercises at home, at work, or at school, without the help of experts, by enabling the patients to check their own COP easily.

Huiting Qiao et al. work with the methods of detection and estimation of the leakage are established and validated. Ventilation experiments are performed, based on the established experimental platform. The airflow and pressure are detected at different locations of the airway to determine the relationship between the leakage and the other variables. They conclude that according to the validation results, the precision of the above-mentioned leakage estimation methods proves to be high. The results lay the foundation for leakage control and compensation in noninvasive ventilation (NIV).

Jae-hoon Heo et al. evaluate the effects of age and gender on the thresholds of perception and muscle response in response to support surface rotation. The results imply that both sensitivities of proprioception in subcortical and cortical levels are deteriorated by aging and that the proprioception sensitivity of cortical control is lower in females than in males in case of the elderly. Inferior perception sensitivity of platform rotation in elderly women may be associated with inferior performance in cortical postural control and a greater fall ratio compared to elderly men, which suggests the need for proprioception trainings.

Chih-Sheng Lin et al. survey and explore the correlation between triggered timing for head and neck CT angiography (TT CTA) scanning and the average of CT values of both left and right arterial to upper sinuses (LRA/US) reaching a maximal ratio using the inverse problem algorithm according to a six-factor semiquantitative analysis of 251 patients. The IPA-based LRA/US analysis is conducted based on clinical data of 251 patients with ischemic stroke symptoms. Six risk factors are used to identify a nonlinear first-order regression correlation between projected and actual LRA/US cases and derive the respective twenty-two terms of the equation. The propose algorithm is considered quite instrumental in predicting the LRA/US with ischemic stroke symptoms in the CTA examination.

Generally, this special selection pursues to discussing the ongoing and active research on Mechanical Engineering Applied to Biomedicine, providing valuable insights and groundbreaking discoveries on updated Human Mechanics ${ }^{1,2,8,12,13,17,18}$ including rehabilitation engineering, finite element analysis and biofluid mechanics, etc. as well as Biomedical Imaging. ${ }^{3-7,9-11,14-16,19,20}$ We hope that the selected papers will provide the readers with useful examples of state-of-art research on the 
most outstanding frameworks in mechanical engineering used for biomedicine and their application to challenge biomedical problems.

Guest Editors

Esteban Peña Pitarch Universitat Politècnica de Catalunya (UPC), Spain

Agnès Drochon Centre National de la Recherche Scientifique (CNRS), France

Eddie Y. K. Ng

Nanyang Technological University, Singapore

\section{References}

1. Liu F, Ng EYK, Zi Chen, A special section on biological mechanics, J Mech Med Biol 15(6):1502002-1-1502002-3, 2015.

2. Liu F, Ng EYK, A special section on biological mechanics, $J$ Mech Med Biol 16(8):1602002-1-1602002-4, 2016.

3. Liu F, Ng EYK, A special section on biomedical imaging in diagnosis and treatment (Part 1), J Med Imag Health Inform 6(5):1209-1211, 2016.

4. Liu F, Ng EYK, A special section on biomedical imaging in diagnosis and treatment (Part 2), J Med Imag Health Inform 16(7):1670-1672, 2016.

5. Liu F, Ng EYK, A special section on biomedical imaging in diagnosis and treatment (Part 3), J Med Imag Health Inform 17(1):126-128, 2017.

6. Liu F, Ng EYK, A special section on methods and application in biomedical imaging (Part 1), J Med Imag Health Inform 7(5):919-921, 2017.

7. Liu F, Ng EYK, A special section on methods and application in biomedical imaging (Part 2), J Med Imag Health Inform 7(7):1522-1524, 2017.

8. Liu F, Ng EYK, A special section on biological mechanics, J Med Imag Health Inform 17(7):1702002-1-1702002-7, 2017.

9. Liu F, Ng EYK, A special section on methods and application in biomedical imaging (Part 3), J Med Imag Health Inform 8(1):1-4, 2018.

10. Gomez L, Ng EYK, A special section on methods and application in biomedical imaging (Part 1), J Med Imag Health Inform 8(7):1364-1367, 2018.

11. Gomez L, Ng EYK, A special section on methods and application in biomedical imaging (Part 2), J Med Imag Health Inform 8(8): 1607-1610, 2018.

12. Peña E, Drochon A, Ng EYK, A special selection on biological applications of mechanics, J Mech Med Biol 18(7):1802001-1-1802001-8, 2018.

13. Peña E, Drochon A, Ng EYK, A special selection on biological applications of mechanics, J Mech Med Biol 18(8):1802002-1-1802002-8, 2018.

14. Gomez L, Ng EYK, A special section on methods and application in biomedical imaging (Part 3), J Med Imag Health Inform 9(1):43-46, 2019.

15. Gomez L, Ng EYK, A special section on methods and application in biomedical imaging (Part 1), J Med Imag Health Inform 9(7): 1415-1417, 2019.

16. Gomez L, Ng EYK, A special section on methods and application in biomedical imaging (Part 2), J Med Imag Health Inform 9(9):1849-1852, 2019.

17. Peña E, Drochon A and Ng EYK, A special selection on biomechanics in medical application - (Part 1), J Mech Med Biol 19(7):1902003-1-1902003-8, 2019. 
18. Peña E, Drochon A and Ng EYK, A Special Selection on Biomechanics in Medical Applications - (Part 2), J Mech Med Biol 19(8):1902004-1-1902004-8, 2019.

19. Gomez L, Ng EYK, A special section on emerging techniques for biomedical imaging (Part 3), J Med Imag Health Inform 10(3):610-613, 2020.

20. Gomez L, Ng EYK, A special section on emerging techniques for biomedical imaging (Part 1), J Med Imag Health Inform 10(11):2660-2662, 2020.

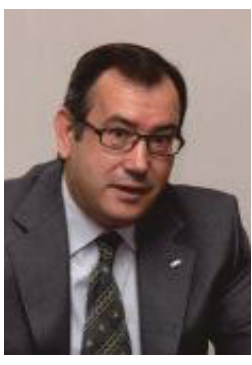

Esteban Peña Pitarch holds a doctorate in the UPC. He has carried out his teaching work at the Technical College of Manresa (EPSEM), since 1988 and belongs to the department of mechanical engineering. He collaborates with the Institute of Industrial and Control Engineering (IOC), UPC, since 2008, in the robotics division. His research is focused on rehabilitation and simulation of stroke survivors, the creation of medical devices and the application of kinematics and dynamics to the human body by way of mathematical tools used in robotics. He manages a group with doctors specialized in physical medicine, rehabilitation, and engineers from a number of different fields. This group has published articles in magazines and congresses and owns two patents relating to medical apparatus. Esteban Peña Pitarch belongs to the Service and Industrial Robotics (SIR) research team and is currently working on two competitive projects as the main researcher of one and collaborating in the other. He is a professor and ex-dean of college Escola Politècnica Superior d'Enginyeria de Manresa (EPSEM) at the Universitat Politècnica de Catalunya (UPC). His teaching expertise is in Kinematics and Dynamics, and Machinery Design for Undergraduate and Graduate degree for more than 30 years. He has two patents and more than 80 papers in international journals and conferences. Research interests are in Virtual Human Modeling, Rehabilitation, and Human Exoskeleton Construction.

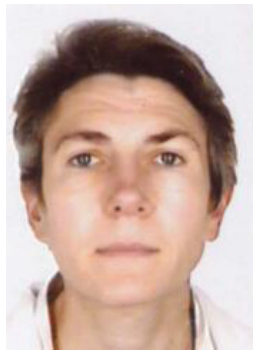

Agnès Drochon is born in 1965, in Châteauneuf-sur-Charente, France. She obtained her diploma of Engineer in mechanics and fluid mechanics at ENSEEIHT (Toulouse, France) (1987), and a $\mathrm{PhD}$ in Biomedical Engineering at the University of Technology of Compiègne (France), in 1991. She is CNRS research fellow since this time, in the Laboratory BMBI ("Biomechanics and Bioengineering") of UTC. Her topics of research are cardio-vascular biomechanics, cells biomechanics, magnetohydrodynamic flow of blood, and related applications. She teaches mathematics and fluid mechanics. 


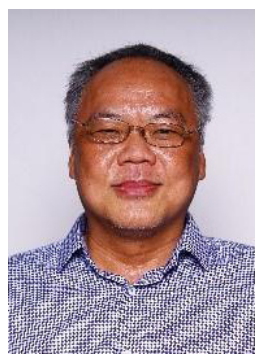

Eddie Y. K. Ng obtained Ph.D. at Cambridge Univiversity and elected as a Fellow of The American Society of Mechanical Engineers [USA]; The Institution of Engineering and Technology [UK], and International Engineering \& Technology Institute $[\mathrm{HK}]$. He researches numerical simulation in the biomedical engineering, thermal-fluids, and health-related diagnosis fields. He is the Editor-in-Chief for 2 ISI-journals which are captured by the JCR within 2-years of their inauguration. He has been recognized internationally for academic excellence. He received numerous best papers, service awards and has graduated 24 Ph.D. and 28 Master students. He is awarded the SPRING-Singapore Merit Award for his work in thermal imagers to screen SARS fever and contributions to the Singapore Standardization Program. Twenty-one of his papers have been adopted as references in Singapore Standard (SS-582, Parts 1\&2: 2020) and ISO/IEC 80601-2-59: 2017. He serves as a panel member for the Singapore Biomedical and Health Standards Committee since 2011. Being a co-inventor of 3 US patents on software classifiers to identify the different stages of breast cancer development in iTBra-system, he is accoladed with equity in a listed company. His ongoing work on non-contact screening for carotid artery stenosis and superficial vein-finder has resulted in 3 TDs. He has notable citations in the field of infrared physics \& technology.

His ORCID is: http://orcid.org/0000-0002-5701-1080 